\title{
Multiscale Hierarchical Micro- and Nanostructures: Nanotubes and Micro-Assembly
}

\author{
Dmitry V. Bavykin, Frank. C. Walsh \\ Energy Technology Research Group, Faculty of Engineering and the Environment, \\ University of Southampton, United Kingdom \\ D.Bavykin@soton.ac.uk
}

Nanostructured materials have attracted a great attention last years due to their unusual physico-chemical properties and potential use in many applications. As a result, our knowledge of synthetic routes and the methods for controlling morphology, shape and the geometry of individual nanostructures (including nanotubes, nanofibers, nanowires, nanosheets etc) has significantly improved. Early attempts to use nanostructured materials in many technological application has shown the shortage of the methods, which could allow facile packing of nanostructures into the various micrometer size structures with defined geometry and dimensions. Such control of the morphology simultaneously in both micro- and nanoscale, although very common in natural biological materials, is very challenging task for artificial synthetic materials.

General overview of several approaches for crafting multiscale hierarchical micro- and nanostructures including both top down and bottom up methodologies as well as their combinations is to be discussed. Examples of simultaneous assembly of mictro and nanotubes of $\mathrm{TiO}_{2}$ [1], step by step crafting of titanate nanotubes into $\mathrm{TiO}_{2}$ nanotubes [2] or on the surface of $\mathrm{ZnO}$ nanorods [3] are to be considered.

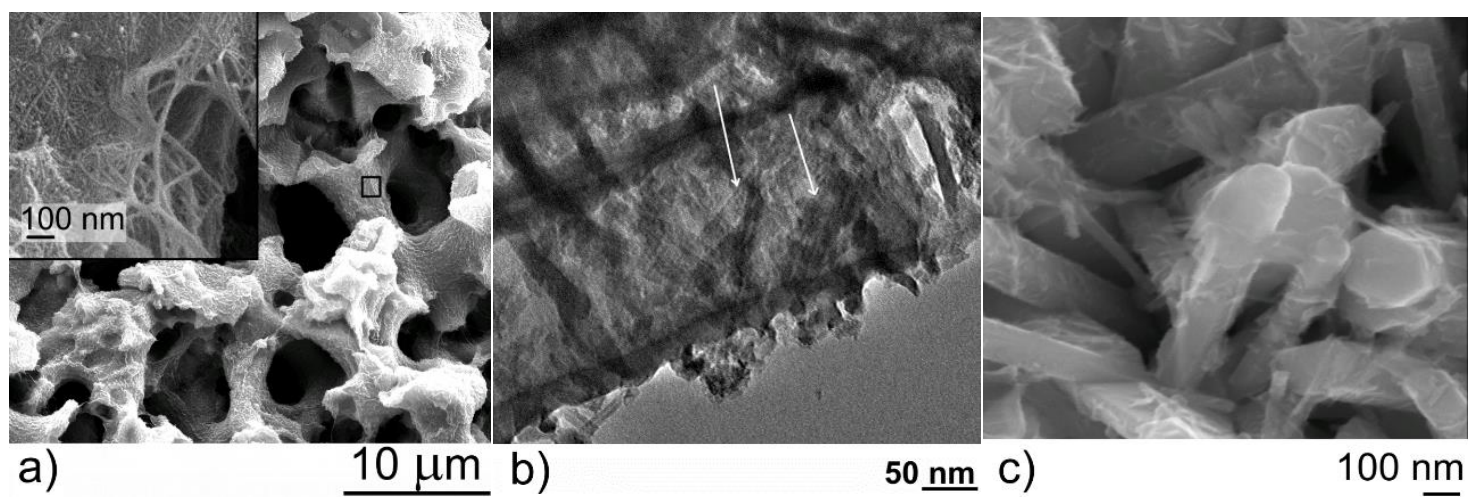

Fig. 1: (a) SEM image of spontaneously formed hierarchical assembly of titanate nanotubes from [1], (b) TEM image of titanate nanotubes inside anodic $\mathrm{TiO}_{2}$ nanotubes [2], and (c) $\mathrm{ZnO}$ nanorods array decorated with titanate nanotubes [3].

The needs and benefits of such multiscale micro- and nanometres hierarchical structures in several technological applications including dye sensitized solar cells, fuel cells and lithium batteries, catalysis will be illustrated in terms of transport, capacity and cost.

\section{References}

[1] D.V. Bavykin, A.N. Kulak, and F.C. Walsh, Langmuir, vol. 27, pp. 5644-5649, 2011.

[2] D.V. Bavykin, L. Passoni, and F.C. Walsh, Chem. Comm., vol. 49, no. 62, pp. 7007-7009, 2013.

[3] J.S. de Souza, W.M Carvalho-Jr, F.L. Souza, C. Ponce de Leon, D.V Bavykin, and W.A. Alves. (2015, December 01). J. Mater. Chem. A [Online]. Available: http://dx.doi.org/10.1039/C5TA06646H. 